\title{
DESARROLLO Y VALIDACIÓN DE UN MÉTODO DE CALIBRACIÓN DE ANALIZADORES DE GASES PARA VEHÍCULOS CON MOTORES DE ENCENDIDO POR CHISPA
}

\begin{abstract}
Resumen
De todo el conjunto de aspectos fundamentales de la revisión técnica de un vehículo automotor, la determinación de las proporciones de gases contaminantes emitidos es crítica, puesto que una gran cantidad de los vehículos que operan en Costa Rica no cumple con los límites establecidos por la Ley de tránsito por vías públicas terrestre $N^{\circ} 7331$ de Costa Rica (1993), en especial aquellos con tecnologías menos modernas. En vista de las implicaciones legales asociadas, la calibración adecuada de los equipos analizadores de gases resulta fundamental para asegurar la veracidad de las mediciones que se realizan en las estaciones de revisión de carácter oficial.

El Laboratorio de Metrología, Normalización y Calidad (LABCAL) de la Universidad de Costa Rica (UCR), desarrolló un método de calibración de estos equipos basándose en los principios de calibración multipunto, para lo cual se utilizan tres cilindros con mezclas de los gases medidos en tres concentraciones diferentes. Mediante el proceso de validación se determinó que los procedimientos de laboratorio usados para las calibraciones según lo requiere el principal cliente nacional, son tales que se puede asegurar que no existe variación significativa entre las mediciones realizadas por distintos operadores, con un nivel de confianza del $95 \%$.
\end{abstract}

Palabras claves: calibración, analizadores de gases, inspección técnica vehicular.

\begin{abstract}
The measurement of internal combustion engine exhaust gas composition during legally required technical vehicle inspection is critical due to the large amount of vehicles that fall outside Costa Rican emissions law limits, especially mainly those with previous-generation fuel metering technologies. Therefore, it is fundamental to assure that all instrumentation associated to official measurements is as accurate as possible with its inherent metering technology.

The LABCAL at the Universidad de Costa Rica has developed a calibration method based on multipoint calibration, in which cylinders with three different gas concentrations were used.

Such validation process showed that the procedures used were acceptable to the conditions and specifications of the major client, and in fact it is demonstrated that there is no significant variation between different instrument operation personnel, with a reliability level of $95 \%$.
\end{abstract}

Keywords: calibration, exhaust gas analyzer, vehicle acceptability.

Recibido: 20 de enero del 2009 • Aprobado: 17 de marzo del 2009.

\section{INTRODUCCIÓN}

Debido a los efectos sensibles de la contaminación ambiental por el uso de los combustibles fósiles, se ha creado conciencia en los políticos y en la sociedad en general para disminuir los niveles de emisiones de gases contaminantes a la atmósfera. En Costa Rica el sector transporte es el principal contribuyente a este problema, tal como se plantea en el Plan Nacional de Energía 2008-2013, elaborado por la Dirección Sectorial de Energía (DSE, 2008).
Por lo anterior, se incluyó la medición y la regulación de la emisión de gases de combustión para los vehículos con motores de encendido por chispa como parte fundamental de la Revisión Técnica Vehicular (RTV). La RTV se aplica en Costa Rica desde el año 2002 y está regulada por el Ministerio de Obras Públicas y Transportes (MOPT) y ejecutada por la empresa Riteve SyC, S. A. (RITEVE, 2007) a la cual el MOPT le delegó dicha tarea.

La prueba de emisión de gases es crítica dentro de la RTV, pues gran cantidad de vehículos 
sobrepasa los valores mínimos de emisión de monóxido de carbono ( $\mathrm{CO}$ ), hidrocarburos no quemados $(\mathrm{HC})$ y dióxido de carbono $\left(\mathrm{CO}_{2}\right)$ establecidos en la Ley General de Tránsito por Vías Terrestres $N^{\circ} 7331$ de Costa Rica de 1993.

Durante el segundo semestre del 2004, en el Laboratorio de Metrología Normalización y Calidad (LABCAL) de la Universidad de Costa Rica (UCR), se inició un proceso de desarrollo y planteamiento de un método de calibración para analizadores de gases de los vehículos que utilizan motores de encendido por chispa.

En este documento se describirán el planteo, la experimentación y la validación del método de calibración para analizadores de gases desarrollado en LABCAL.

\section{DESARROLLO DEL MÉTODO DE CALIBRACIÓN DE ANALIZADORES DE GASES}

El desarrollo del método constó de una serie de etapas que se describen a continuación.

\subsection{Investigación preliminar y planteamiento inicial}

Seestudiaronnormasinternacionalesrelacionadas con la calibración de analizadores de gases para vehículos con motores de encendido por chispa (EPA, 1993; OIML, 2000) y los requerimientos del cliente establecidos en los manuales del fabricante de sus equipos (MAHA, 2001), entre otros.

El conocimiento del equipo analizador de gases y el software de interfase utilizado, también se llevó a cabo en esta etapa preliminar. Adicionalmente, se analizaron las características técnicas del mismo: rango de medición, gases de medición, condiciones de operación y principio de funcionamiento.

\subsection{Experimentación y pruebas}

Sehicieronunaseriedepruebas paradeterminar la influencia en las mediciones de factores como: tiempo de respuesta y estabilización de los sensores, tiempo de realización de las pruebas, técnicos y personal que realizarían la prueba, número de repeticiones, comportamiento general de los equipos y del montaje experimental. Se consideró que para la calibración de los analizadores de gases se haría pasar por el sensor una mezcla de gases con concentraciones certificadas de cada componente.

\subsection{Cálculo del error de indicación y estimación de la incertidumbre de medición}

Se determinó el procedimiento de cálculo del error de indicación de los analizadores de gases. Se hizo el cálculo para cada uno de los componentes de la mezcla y en cada una de las concentraciones evaluadas. Además, se identificaron las fuentes de incertidumbre incluyendo las que generan evaluación de la incertidumbre tipo A y tipo B (BIPM, IEC, IFCC, ISO, IUPAC, IUPAP \& OIML, 1995).

\subsection{Validación del método}

Para cada método de calibración que se desarrolla en el LABCAL, se sigue un proceso de validación para demostrar la competencia técnica del Laboratorio en el uso del método. Este proceso está basado en la norma INTEISO 5725-2 (INTECO, 2006). Además se incluye el cálculo de dos factores: índice de idoneidad $\left(\mathrm{I}_{i d}\right)$ y relación de exactitud (RE), así como la determinación de la mejor capacidad de medición (MCM) del Laboratorio (METAS y METRÓLOGOS ASOCIADOS, 2006).

En la validación del método participaron cuatro técnicos, y cada uno de ellos realizó cuatro calibraciones que permitieron determinar la repetibilidad y reproducibilidad del método.

\subsection{1 Índice de idoneidad}

El índice de idoneidad es un parámetro basado en el concepto de índice de consistencia (METAS y METRÓLOGOS ASOCIADOS, 2002), el cual es una relación entre la incertidumbre de medición del Laboratorio y la incertidumbre requerida. Se utiliza comúnmente en procesos 
de control de calidad. En LABCAL se define el índice de idoneidad de la siguiente manera:

$$
\mathrm{I}_{\mathrm{id}}=\frac{\mathrm{u}_{\mathrm{req}}(\mathrm{y})}{\mathrm{u}_{\mathrm{C}}(\mathrm{y})}
$$

Donde:

$\mathrm{u}_{\text {req }}(\mathrm{y}): \quad$ Incertidumbre requerida $(\mathrm{cmol} / \mathrm{mol}) \mathrm{o}$ $(\mu \mathrm{mol} / \mathrm{mol})$

$\mathrm{u}_{C}(\mathrm{y}): \quad$ Incertidumbre estándar combinada de medición $(\mathrm{cmol} / \mathrm{mol})$ o $(\mu \mathrm{mol} / \mathrm{mol})$

Si el $\mathrm{I}_{\text {id }} \geq 1$ se considera que el Laboratorio tiene la capacidad para cumplir los requerimientos metrológicos del cliente, si por el contrario, el índice es menor que 1, entonces el sistema de calibración no se considera apropiado para cumplir con los requerimientos de incertidumbre.

\subsubsection{Relación de exactitud}

Según la norma ISO 10012-1 se ha definido este concepto de relación de exactitud (TAR, Traceability Accuracy Ratio) (ISO, 1992), como una relación entre la exactitud del equipo y la exactitud del patrón. Por lo tanto, en el LABCAL se ha hecho la determinación de esta relación considerando la incertidumbre requerida y la incertidumbre del patrón, tal como se muestra en la ecuación (2).

$\mathrm{RE}=\frac{\mathrm{u}_{\mathrm{req}}(\mathrm{y})}{\mathrm{u}_{\mathrm{P}}(\mathrm{y})}$

Donde:

$\mathrm{u}_{\text {req }}(\mathrm{y}) \quad$ Incertidumbre requerida $(\mathrm{cmol} / \mathrm{mol}) \mathrm{o}$ $(\mu \mathrm{mol} / \mathrm{mol})$

$\mathrm{u}_{P}(\mathrm{y})$ : Incertidumbre expandida del patrón $(\mathrm{cmol} / \mathrm{mol})$ o $(\mu \mathrm{mol} / \mathrm{mol})$

Se recomienda que la RE sea mayor que 3 para asegurar que los patrones disponibles utilizados sean adecuados para cumplir con los requerimientos metrológicos.

\subsubsection{Mejor capacidad de medición}

La mejor capacidad de medición de un laboratorio se refiere a la mínima incertidumbre que se puede lograr durante las calibraciones rutinarias; en el caso del LABCAL se determina con la ecuación (3).

$$
\mathrm{e}_{\mathrm{L}}=\overline{\mathrm{C}_{\mathrm{c}}}-\mathrm{C}_{\mathrm{p}}
$$

Donde:

$\mathrm{U}_{p}$ : Incertidumbre expandida con un nivel de confianza del $95 \%$ para un factor de cobertura $\mathrm{k}_{P}$ determinado para el número de grados de libertad definido.

\subsection{Otros aspectos considerados asociados al sistema de gestión de la calidad de LABCAL}

El Laboratorio posee un Sistema de Gestión de la Calidad según la norma INTE-ISO/IEC 17025:2005 (INTECO, 2005); el método descrito en este artículo está incluido en el alcance del mismo.

Parte de las principales actividades realizadas fueron: evaluación de los proveedores de los cilindros con la mezcla de gas, validación del procedimiento de calibración, evaluación del desempeño del personal, además de un control de la calidad de las mediciones.

\section{RESULTADOS}

En esta sección se presentan los resultados de cada una de las etapas del desarrollo de los procedimientos.

\subsection{Investigación preliminar y planteamiento inicial}

Los equipos analizadores de gases constan de sensores para cada gas que tienen como principio de funcionamientola absorción infrarroja de gases. 
Específicamente, un haz de radiación infrarrojo se hace pasar por la cámara de medición del equipo; según sea el tipo de gas y la concentración presente en la cámara, el haz infrarrojo se va modificando de una manera característica para cada tipo de gas, y dicha variación es registrada en los sensores eléctricos.

Con base en lo anterior, se decidió utilizar el principio de calibración multipunto para tres concentraciones de gases dentro del rango de medición. Se utilizaron tres cilindros diferentes, cada uno de ellos con una concentración específica de $\mathrm{CO}, \mathrm{CO}_{2}$ y $\mathrm{HC}$ (el equipo de medición considera como hidrocarburos no quemados al propano, $\mathrm{C}_{3} \mathrm{H}_{8}$ ). En el Cuadro 1 se presentan las concentraciones de cada gas recomendadas para la calibración, así como la máxima incertidumbre recomendada por la investigadora. Se debe aclarar que esta recomendación de máxima incertidumbre se obtiene de la validación del método que se presentará en el apartado 3.4 de este documento.

\subsection{Experimentación y pruebas}

De la etapa de pruebas y experimentación, se definieron los siguientes parámetros significativos en el marco del método propuesto:

- Tiempo de espera para estabilización de los sensores de medición de cada componente de la mezcla de gas: $60 \mathrm{~s}$.

- Número de repeticiones por cada concentración: cinco por cada componente de la mezcla de gas.
- Tiempo entre cada repetición: $10 \mathrm{~s}$.

- Tiempo total de calibración para cada equipo: de $45 \mathrm{~min}$ a $60 \mathrm{~min}$.

Además, se pudo evaluar la habilidad de los técnicos y del personal autorizado para realizar la calibración, determinándose que no existe diferencia estadística significativa entre las mediciones que realiza cada uno de ellos.

Se establecieron las medidas básicas de seguridad que se deberán acatar durante la realización de las calibraciones: uso de mascarillas en espacios cerrados, presencia de un extintor funcional, ventilación suficiente, y un manejo adecuado de las válvulas de suministro y regulación de la mezcla de gases.

\subsection{Cálculo del error de indicación y estimación de la incertidumbre de medición}

\subsubsection{Error de indicación}

El error de indicación se determinó mediante la ecuación (4).

$$
\mathrm{e}_{\mathrm{L}}=\overline{\mathrm{C}_{\mathrm{c}}}-\mathrm{C}_{\mathrm{p}}
$$

Donde:

$\overline{\mathrm{C}}_{\mathrm{C}}$ : Promediodelosvalores deconcentración medidos en el equipo, $(\mathrm{cmol} / \mathrm{mol})$ o $(\mu \mathrm{mol} / \mathrm{mol})$

Cuadro 1. Características del gas patrón.

\begin{tabular}{cccccc} 
GAS & Baja & Media & Alta & $\begin{array}{c}\text { Máxima } \\
\text { incertidumbre } \\
\text { permitida }\end{array}$ & Unidades \\
CO & $4-8$ & $10-12$ & $14-18$ & 0,1 & $\mathrm{cmol} / \mathrm{mol}$ \\
$\mathrm{CO} 2$ & $1-2$ & $4-6$ & $8-10$ & 0,1 & $\mathrm{cmol} / \mathrm{mol}$ \\
$\mathrm{HC}$ & $100-200$ & $200-500$ & $1000-3000$ & 45 & $\mu \mathrm{mol} / \mathrm{mol}$ \\
& \multicolumn{5}{c}{ Fuente: (La autora). }
\end{tabular}


$\mathrm{C}_{p}$ : Valor de concentración del gas patrón, $(\mathrm{cmol} / \mathrm{mol})$ o $(\mu \mathrm{mol} / \mathrm{mol})$

$\mathrm{e}_{L}$ : $\quad$ Error de indicación del instrumento de medición, $(\mathrm{cmol} / \mathrm{mol})$ o $(\mu \mathrm{mol} / \mathrm{mol})$

3.3.2 Estimación de la incertidumbre de medición

Las fuentes de incertidumbre identificadas son:

- La trazabilidad del material de referencia certificado $\left(\mathrm{u}\left(\Delta \mathrm{e}_{P}\right)\right)$.

- La resolución del equipo $\left(\mathrm{u}\left(\Delta \mathrm{e}_{r e s}\right)\right)$.

- La tolerancia reportada por el fabricante del equipo de medición debida a la deriva $\left(\mathrm{u}\left(\Delta \mathrm{e}_{\text {deriva }}\right)\right)$.

- La variabilidad de los datos obtenida por la repetición de pruebas. $\left(\mathrm{u}\left(\Delta \mathrm{e}_{\text {rep }}\right)\right)$

Por lo tanto, la incertidumbre combinada de medición se expresó mediante la ecuación (5).

Y la incertidumbre expandida con la ecuación (6).

$\mathrm{U}_{\mathrm{P}}=\mathrm{k}_{\mathrm{P}} \cdot \mathrm{u}_{\mathrm{c}}\left(\mathrm{e}_{\mathrm{L}}\right)$

Con $\mathrm{k}_{P}$ como factor de cobertura para un nivel de confianza del $95 \%$.

En el apartado 2.4.3 se presentaron los resultados de la MCM, que corresponden a la incertidumbre expandida para cada uno de los componentes de la mezcla de gas y para cada concentración evaluada.

\subsection{Validación del método}

En el Cuadro 2 se presentan los resultados obtenidos de la validación del método de calibración de analizadores de gases, según los criterios del LABCAL y descritos anteriormente en la sección 2.3 de este documento.

\subsubsection{Normalidad de los datos}

Los datos obtenidos de las calibraciones realizadas como parte de la validación, mostraron un comportamiento normal, tal como se puede observar en la Figura 1.

\subsubsection{Fuentes de incertidumbre y su peso en la incertidumbre combinada}

Se determinó el peso de cada una de las fuentes de incertidumbre en relación con la incertidumbre combinada, lo que se muestra en la Figura 2. Es evidente que no existe un comportamiento uniforme entre los gases en todas las concentraciones.

Por ejemplo, la repetibilidad resulta considerableenelcasodetodaslasconcentraciones de $\mathrm{CO} 2$, y en las concentraciones baja y media de $\mathrm{C} 3 \mathrm{H} 8$, no así para el resto.

3.4.3 Determinación de los grados efectivos de libertad y el factor de cobertura

De las fuentes de incertidumbre identificadas y los respectivos grados de libertad de cada una según el tipo de distribución, se obtuvieron valores de grados efectivos de libertad que implican un factor de cobertura de 2, para una confiabilidad del $95 \%$.

\subsection{Otros aspectos considerados para la validación del método}

Como parte de la evaluación de proveedores de mezclas de gases se determinó que en el país no existen empresas que realicen las mezclas directamente, sino que las empresas vendedoras deben importarlo directamente de las casas matrices o de laboratorios en otros países; por lo tanto, los aspectos que se consideraron en la evaluación fueron:

- Trazabilidad de los métodos, expresada con la incertidumbre de cada componente de la mezcla.

$\mathrm{u}_{\mathrm{c}}\left(\mathrm{e}_{\mathrm{L}}\right)=\sqrt{\mathrm{u}^{2}\left(\Delta \mathrm{e}_{\text {res }}\right)+\mathrm{u}^{2}\left(\Delta \mathrm{e}_{\mathrm{p}}\right)+\mathrm{u}^{2}\left(\Delta \mathrm{e}_{\mathrm{rep}}\right)+\mathrm{u}^{2}\left(\Delta \mathrm{e}_{\text {deriva }}\right)}$ 
- Tiempo de entrega.

- Disponibilidad de las concentraciones requeridas.

Por otro lado, la evaluación del personal incluyó un análisis de repetibilidad del método utilizando como fuente los técnicos que participaron en la validación; en este análisis se demostró que no existe variabilidad significativa entre las mediciones que realiza cada metrólogo, probándose de esta manera la competencia técnica de cada uno.

Cuadro 2. Resultados de la validación del método.

\begin{tabular}{|c|c|c|c|c|c|c|}
\hline Gas & Concentración & $\begin{array}{l}\text { Índice de } \\
\text { idoneidad }\end{array}$ & $\begin{array}{c}\text { Relación de } \\
\text { exactitud }\end{array}$ & Valor patrón & MCM & Unidades \\
\hline \multirow{3}{*}{$\mathrm{CO}$} & Baja & 1,6 & 6 & 0,506 & 0,021 & $\mathrm{cmol} / \mathrm{mol}$ \\
\hline & Media & 2,8 & 6 & 1,001 & 0,024 & $\mathrm{cmol} / \mathrm{mol}$ \\
\hline & Alta & 4,8 & 6 & 3,490 & 0,048 & $\mathrm{cmol} / \mathrm{mol}$ \\
\hline \multirow{3}{*}{$\mathrm{CO} 2$} & Baja & 1,2 & 6 & 8,019 & 0,460 & $\mathrm{cmol} / \mathrm{mol}$ \\
\hline & Media & 1,8 & 6 & 10,02 & 0,37 & $\mathrm{cmol} / \mathrm{mol}$ \\
\hline & Alta & 2,2 & 6 & 14,00 & 0,43 & $\mathrm{cmol} / \mathrm{mol}$ \\
\hline \multirow{3}{*}{ С3H8 } & Baja & 3,0 & 6 & 101,0 & 2,3 & $\mu \mathrm{mol} / \mathrm{mol}$ \\
\hline & Media & 4,7 & 6 & 301,0 & 4,2 & $\mu \mathrm{mol} / \mathrm{mol}$ \\
\hline & Alta & 5,3 & 6 & 996,0 & $\begin{array}{c}4,2 \\
12,6\end{array}$ & $\mu \mathrm{mol} / \mathrm{mol}$ \\
\hline
\end{tabular}

Fuente: (La autora).
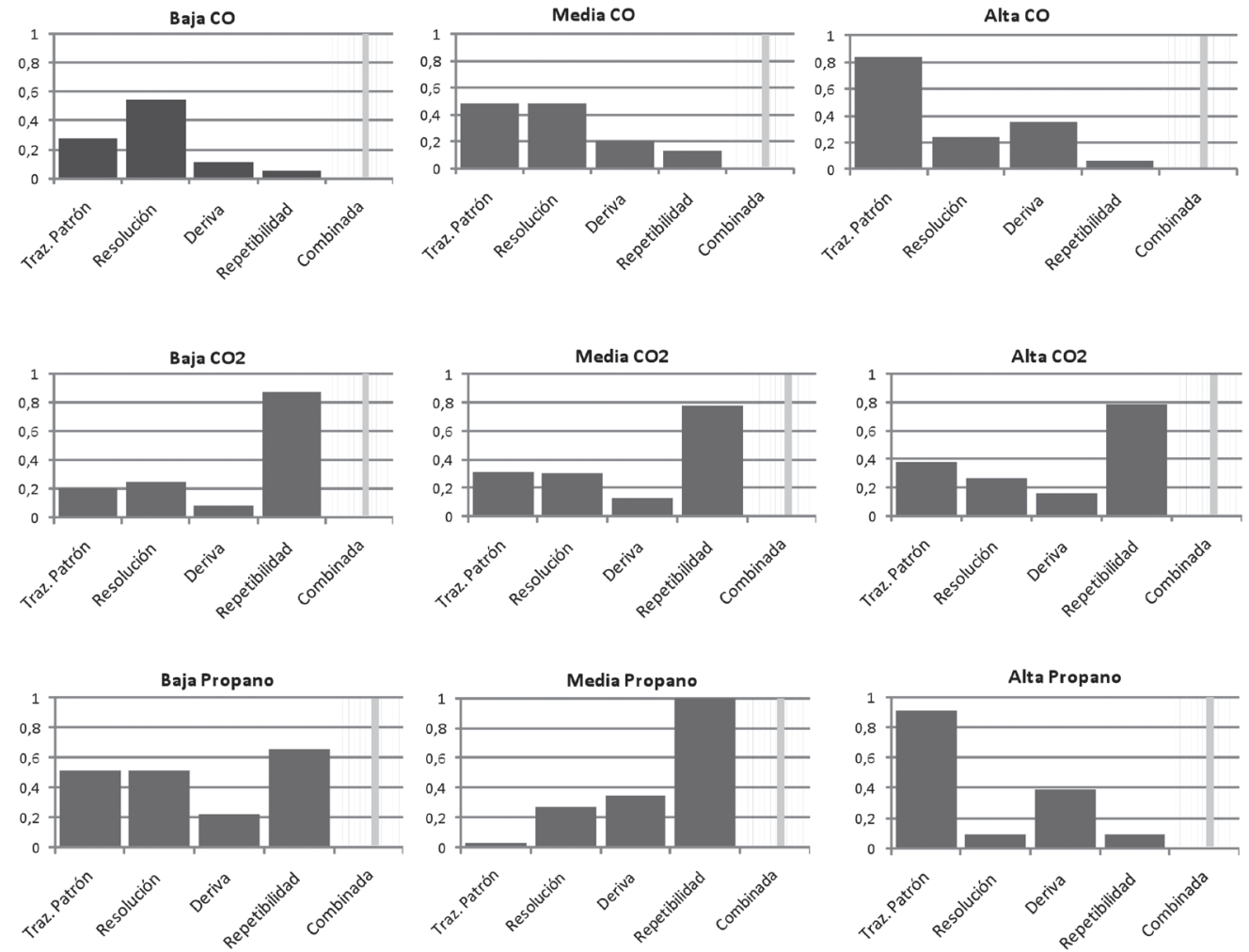

Figura 2. Peso de las fuentes de incertidumbre respecto a la incertidumbre combinada.

Fuente: (La autora). 


\section{DISCUSIÓN Y CONCLUSIONES}

El desarrollo de este método de calibración de analizadores de gases para vehículos de encendido por chispa ha generado una importante experiencia profesional tanto para el LABCAL, como para los investigadores involucrados en su desarrollo al convertirse el Laboratorio en el único del país que realiza este tipo de calibraciones.

Los resultados de la validación demostraron que el LABCAL tiene la competencia técnica para realizar las calibraciones a los analizadores de gases. Se observa del valor de índice de idoneidad mayor que 1 para todos los casos.

Aunque la trazabilidad de las mezclas de gases utilizadas representa una fuente importante por considerar para el cálculo de incertidumbre del método, se puede observar de los resultados de relación de exactitud, que los patrones utilizados son adecuados para los requerimientos metrológicos del cliente y los establecidos en la normativa internacional (OIML, 2000).

De la validación también se puede comprobar que las fuentes de incertidumbre consideradas representan satisfactoriamente los errores tipo A y tipo B, por lo tanto se demuestra la competencia del LABCAL para la calibración de analizadores de gases.

\section{AGRADECIMIENTOS}

- Al Ing. Raziel Farid Sanabria Sandí, quien participó activamente en todas las etapas del desarrollo del método.

- A la empresa Riteve SIC, S. A. por la colaboración brindada durante el desarrollo y la validación del método.

\section{REFERENCIAS BIBLIOGRÁFICAS}

BIPM, IEC, IFCC, ISO, IUPAC, IUPAP \& OIML. (1995). Guide to the expression of uncertainty in measurement. ( $1^{\text {a }}$ ed.) International Organization for Standardization: Switzerland
Dirección Sectorial de Energía (DSE). (2008). Plan nacional de Energía 2008-2013. San José. Extraído de http://www.dse. go.cr/es/03 publicaciones/01 politicaenerg/ VplanNacionalEnergia.pdf

EPA (United States Environmental Protection Agency). (1993). Method 205. Verification of gas dilution system for field instrument calibration. United States of America.

INTECO. (2005). INTE- ISO/IEC 17025:2005. Requisitos generales para la competencia de laboratorios de ensayo y calibración. (1a ed.) San José: Editor.

INTECO. (2006). INTE-ISO 5725-2:2006 Exactitud (veracidad y precisión) de resultados y métodos de precisión - Parte 2: método básico para la determinación de la repetibilidad y reproducibilidad de un método de medición normalizado. ( $1^{\mathrm{a}}$ ed.). San José: Editor.

International Organization for Standardization (ISO).(1992).ISO10012-1.Qualityassurance requirements for measuring equipment Part 1: Metrological confirmation system for measuring equipment. Geneva, Switzerland.

International Organization of Legal Metrology (OIML). (2000). OIML R 99, Instruments for measuring vehicle exhaust emissions. Third edition: France.

Ley de tránsito por vías públicas terrestres (1993, Abril 13). La Gaceta N. ${ }^{\circ}$ 76. Asamblea Legislativa de Costa Rica.

Maschinenbau Haldenwang $\mathrm{GmbH} \& \mathrm{Co}$ (MAHA). (2001). Manual MGT5 del equipo a calibrar. Alemania.

METAS \& METRÓLOGOS ASOCIADOS. (2002, Octubre). Evaluación de consistencia metrológica. La Guía MetAs, № 10. Extraído de: http://www.metas.com.mx/guiametas/ La-Guia-MetAs-02-10-Consist.pdf. 
METAS \& METRÓLOGOS ASOCIADOS. (2006, Marzo). Capacidad de medición. La Guía MetAs, No 03. Extraído de: http://www. metas.com.mx/guiametas/La-Guia-MetAs06-03-Mejor-Capacidad-de-Medicion.pdf.

RITEVE SYC. (2007). Anuario Riteve SyC. San José. Extraído de: http://www.rtv.co.cr/docs/ anuario2007.pdf

\section{SOBRE LA AUTORA}

\section{Leonora de Lemos Medina}

Ingeniera Mecánica de la Universidad de Costa Rica.
Magister en Ingeniería Mecánica de la Universidad de Los Andes. Bogotá, Colombia. Magister en Ingeniería Mecánica con énfasis en Sistemas Térmicos y de Energía de la Universidad de Costa Rica.

Profesora Adjunta de la Escuela de Ingeniería Mecánica de la Universidad de Costa Rica.

Investigadora del Laboratorio de Metrología, Normalización y Calidad de la Universidad de Costa Rica

Ciudad Universitaria Rodrigo Facio Brenes, San José. Costa Rica.

Teléfonos: (506) 2511-5553, (506) 2511-5576

Facsímil: (506) 2253-4614

Apartado postal: 1748-1000

Correo electrónico: ldelemos@fing.ucr.ac.cr 